\title{
Effet d'un amendement calco-magnésien associé ou non à une fertilisation, sur le cycle biogéochimique des éléments nutritifs dans une plantation d'épicéa commun (Picea abies Karst) dépérissante dans les Vosges
}

\author{
J Ranger, D Mohamed Ahamed, D Gelhaye \\ INRA, centre de Nancy, équipe Cycles biogéochimiques, 54280 Champenoux, France
}

(Recu le 12 jüllet 1993; accepté le 29 novembre 1993)

\begin{abstract}
Résumé - Le dépérissement forestier des années 1980 a montré la complexité des interactions entre les paramètres biologiques, édaphiques, climatiques et sylvicoles. Le rôle du paramètre édaphique a été testé par des expériences de fertilisation in situ qui ont montré à la fois les causes de certains dépérissements observés sur épicéa commun (Picea abies Karst) dans les Vosges et l'efficacité du remède. Le chaulage associé ou non à une fertilisation complète (NPK) produit dans un délai de 2 ans un reverdissement et une refoliation efficace d'arbres très atteints. Une étude quantitative approfondie du fonctionnement minéral d'un écosystème ayant réagi favorablement à un tel amendement calcaire a été entreprise en 1988 dans les Vosges (col du Bonhomme). Le sol du peuplement témoin est très acide et montre un fonctionnement géochimique dominé par le nitrate et l'aluminium. Le sol s'acidifie et ne neutralise pas les protons d'origine interne ou externe (apports atmosphériques) ; cette acidité n'est neutralisée que très profondément dans le sous-sol et le ruisseau drainant le bassin versant est neutre. Les bilans entrées-sorties, malgré leur limite, montrent pour tous les pas de temps un déficit permanent de l'écosystème en $\mathrm{Mg}$, élément mis en cause dans le dépérissement. Le chaulage neutralise l'acidité (en particulier aluminique) et améliore la nutrition en $\mathrm{Ca}$ et $\mathrm{Mg}$ des arbres ; il conduit également à une diminution des nitrates dans les eaux gravitaires. L'addition de fertilisants qui n'améliore pas l'état sanitaire des arbres et qui conduit à une accélération de la déperdition des cations apportés par l'amendement ne semble pas opportun dans cet écosystème. Le rôle du paramètre édaphique dans le dépérissement forestier est très clairement mis en évidence dans cette expérimentation.
\end{abstract}

Picea abies Karst / acidification des sols / solutions du sol / bilan minéral / écosystème / dépérissement / amendement/ fertilisation

Summary - Effect of liming and its association with fertilization on the biogeochemical cycle of nutrients in a declining spruce stand (Picea abies Karst) in the Vosges (France). The forest decline observed in the 1980 s showed a complex interaction between biological, edaphic, climatic and silvicultural parameters. The effects of the edaphic constraints were studied using in situ fertilization experiments, which demonstrated both the origin of some of the forest decline symptoms observed on 
spruce stands (Picea abies Karst) in the Vosges and the efficiency of the treatments applied. Liming restored the green growth and foliation of severely declining trees within 2 years independently of whether it was associated with complete fertilization (NPK). A detailed quantitative study of the mineral function of a spruce ecosystem which reacted positively to liming was set up in an experiment situated at Le Col du Bonhomme (Vosges). The soil is very acidic and its current geochemistry is dominated by nitrate and aluminium. Acidification is still an active process and the soil cannot neutralize acidity from external (atmospheric input) or internal origins. This acidity must be neutralized at depth in the sub-soil since the water in the catchment stream is neutral. Input-output budgets, even if they were not very accurate, always showed a Mg deficit whatever the time-scale considered (seasonal, yearly or over several years). This element is often cited as the cause of forest decline. Liming neutralized the soil acidity, particularly its $\mathrm{Al}$ component, and increased the $\mathrm{Ca}$ and $\mathrm{Mg}$ tree nutrition of the trees; nitrates decreased in the gravitational solutions of the liming treatment. Addition of fertilizers to liming, which did not clearly increase tree health and accelerated the depletion of $\mathrm{Ca}$ and $\mathrm{Mg}$ caused by liming, did not seem suitable in this situation. This experiment demonstrates clearly the role of edaphic parameters in this particular case of forest decline.

Picea abies Karst / soil acidification / soil solutions / nutrient balance / ecosystem / forest decline / liming/ fertilization

\section{INTRODUCTION}

En France, le dépérissement forestier a été observé pour la première fois en 1983. Les principales essences affectées furent le sapin (Abies alba Miller) et l'épicéa commun (Picea abies Karst), en particulier dans les hautes altitudes et sur les sols les plus pauvres. Les principaux symptômes décrits très précisément par Bonneau et Fricker (1985), puis Bonneau et Landmann (1988), consistent en une défoliation et un jaunissement total des aiguilles de 2 ans et plus. Ce dépérissement a des origines complexes, avec une interaction de causes climatiques, édaphiques, biologiques et sylvicoles.

Au plan édaphique, le dépérissement est largement associé à des carences nutritionnelles, en particulier magnésiennes. Des expérimentations ont donc été conçues pour apporter les éléments déficients et neutraliser en partie l'acidité du sol. En 1985, une série de traitements de chaulage associés ou non à des fertilisations ont été mis en place dans les Vosges sur des peuplements de sapin et d'épicéa commun dépérissants. L'objectif était à la fois de revitaliser les peuplements et de démontrer le rôle des carences nutritionnelles dans le dépérissement (Bonneau et al, 1992).

Les résultats présentés ici concernent une de ces expériences dans laquelle l'amélioration de l'état de santé des peuplements a été très nette. Un dispositif permanent d'étude du fonctionnement de l'écosystème a été installé avec les objectifs suivants: i) caractériser les conditions édaphiques de l'écosystème; ii) identifer et quantifier les modifications induites par les traitements; et iii) préciser les relations entre les paramètres nutritionnels et l'état sanitaire des peuplements.

\section{MATÉRIEL ET MÉTHODES}

Le site d'étude est situé en forêt communale de Plainfaing (Vosges) à proximité du col du Bonhomme. L'altitude est de $1100 \mathrm{~m}$, le climat est de type montagnard à influence océanique (température moyenne annuelle : $5^{\circ} \mathrm{C}$, pluviométrie moyenne annuelle : $1550 \mathrm{~mm}$ ).

La roche mère du sol est le granite leucocrate acide du Valtin, dont la composition minéralogique a été décrite par Hameurt (1967) : $36 \%$ de quartz, $55 \%$ de feldspaths ( $33 \%$ de feldspaths potassiques et $22 \%$ de plagioclases) et $6 \%$ de micas $(3,5 \%$ de biotite et $2,5 \%$ de muscovite). Les réserves totales en "bases" de ce granite 
sont très limitées : $0,36 \%$ de $\mathrm{MgO}$ et $0,7 \%$ de $\mathrm{CaO}$.

Le sol est de type podzolique avec un humus de type moder. La texture de la terre fine est sableuse, les éléments grossiers sont abondants, le drainage interne est rapide. C'est un sol acide $\left(\mathrm{pH}=3,4\right.$ en $\mathrm{A}_{1}$ et 4,4 en $\left.\mathrm{C}\right)$, très fortement désaturé, où les "bases" échangeables occupent moins de $10 \%$ de la garniture ionique du complexe d'échange (tableau I).

Les traitements de chaulage simple (traitement CaMg) ou associé à une fertilisation (traitements NPKCaMg) ont été apportés à la surface du sol à l'automne 1985 (tableau II). II s'agit d'une expérience sans répétition des traitements, mais reproduite dans plusieurs situations dans les Vosges (Bonneau et al, 1992).

Cinq profils de sols de chaque traitement ont été prélevés et analysés indépendamment $(C$, $\mathrm{N}, \mathrm{pH}, \mathrm{CEC}$ et garniture ionique du complexe d'échange) ou pour un échantillon moyen (granulométrie, éléments totaux, éléments libres).

Le dispositif de récolte des solutions a été installé pendant l'été 1988 dans 3 des traitements de l'expérience, le témoin, le chaulage (traitement $\mathrm{CaMg}$ ) et le chaulage associé à une fertilisation (traitement NPKCaMg). II s'agit :

- pour les apports atmosphériques de 2 pluviomètres situés sur une tour de $12 \mathrm{~m}$ dans une clairière proche du peuplement, et par traitement ;

- pour les pluviolessivats de 4 gouttières de $2 x$ $0,09 \mathrm{~m}$;

- pour le ruissellement de troncs de 5 "colliers" ceinturant les arbres représentatifs des différentes classes de diamètre ;
- pour les solutions du sol, de 9 mini-lysimètres sans tension de $40 \times 4 \mathrm{~cm}$ introduits sous l'horizon holorganique, et de 3 plaques lysimétriques, sans tension, de $40 \times 30 \mathrm{~cm}$ en polyéthylène, insérées à 15,30 et $60 \mathrm{~cm}$ de profondeur.

Quatre plaques lysimétriques ont été insérées à 1,20 m de profondeur (en deçà de la zone racinaire) dans le traitement temoin. Les eaux d'une source et d'un ruisseau du bassin versant ont également été échantillonnées.

Le suivi lysimétrique a été effectué par des prélèvements mensuels pendant 3 ans, de décembre 1988 à décembre 1991 .

Le peuplement est une deuxième génération d'épicéa commun (Picea abies Karst) de 70 ans, planté après la coupe suite à un chablis d'un peuplement pratiquement de même nature (l'aménagement forestier signale un certain mélange avec du sapin), lui-même introduit sur un recrû forestier succédant à une lande parcourue.

Une évaluation de la biomasse et de l'immobilisation minérale du peuplement a été effectuée à partir d'un échantillon de 4 arbres choisis indépendamment des traitements, considérant que ces derniers appliqués depuis 5 ans ne pouvaient avoir d'influence significative sur la concentration moyenne des compartiments ligneux pris en compte dans le calcul de l'immobilisation.

Les restitutions par les litières ont été évaluées pendant les 3 années de l'étude, à partir de 20 bacs rectangulaires de $45 \times 30 \mathrm{~cm}$ disposés régulièrement sur 2 transects dans chacun des traitements.

Les analyses de sols ont été réalisées conformément aux protocoles définis par Bonneau et

Tableau I. Principales caractéristiques analytiques du sol du col du Bonhomme.

\begin{tabular}{|c|c|c|c|c|c|c|c|c|c|c|c|c|}
\hline \multirow{2}{*}{$\begin{array}{l}\text { Horizon et } \\
\text { profondeur } \\
(\mathrm{cm})\end{array}$} & \multicolumn{3}{|c|}{ Texture (\%) } & \multirow{2}{*}{$\begin{array}{c}\text { Éléments } \\
\text { grossiers } \\
(\%)\end{array}$} & \multirow{2}{*}{$\begin{array}{r}d a \text { a } \\
\left(\mathrm{g} / \mathrm{cm}^{3}\right)\end{array}$} & \multirow{2}{*}{$\begin{array}{l}\text { Corg } \\
(\%)\end{array}$} & \multirow[t]{2}{*}{$C / N$} & \multicolumn{2}{|c|}{ Éléments libres } & \multirow{2}{*}{$\begin{array}{c}\mathrm{pH} \\
\left(\mathrm{H}_{2} \mathrm{O}\right)\end{array}$} & \multirow{2}{*}{$T^{c}$} & \multirow[t]{2}{*}{$S / T^{d}$} \\
\hline & Arg & Lim & Sab & & & & & $\begin{array}{l}\mathrm{Fe}_{2} \mathrm{O}_{3} \\
(\%)^{\mathrm{b}}\end{array}$ & $\begin{array}{l}\mathrm{Al}_{2} \mathrm{O}_{3} \\
(\%)\end{array}$ & & & \\
\hline$A, 0-7$ & 21 & 32 & 47 & 13 & 0,44 & 13,4 & 18 & 0,8 & 0,19 & 3,4 & 15,2 & 14 \\
\hline $\mathrm{A}_{2} \mathrm{Bh} 7-14$ & 15 & 28 & 56 & 17 & 0,63 & 6,4 & 22 & 1,1 & 0,19 & 3,6 & 16,3 & \\
\hline Bh $14-20$ & 13 & 28 & 59 & 17 & 0,6 & 3,7 & 22 & 1,6 & 0,2 & 3,8 & 16,7 & 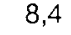 \\
\hline Bs $20-60$ & 11 & 30 & 59 & 20 & 0,67 & 2,7 & 22 & 1,7 & 0,6 & 4,1 & 11,6 & 6,7 \\
\hline$C>60$ & 9 & 31 & 61 & 20 & 0,7 & 2,1 & 23 & 1,6 & 0,64 & 4,4 & 7,8 & 8,8 \\
\hline
\end{tabular}

a Densité apparente de la terre fine; b Fe (Mehra et Jackson, 1960) ; Al (Tamura, 1958); c CEC (effective) : extraction KCl1N (Rouiller et al, 1981); d taux de saturation. 
Tableau II. Traitements appliqués (données en $\mathrm{kg} / \mathrm{ha}$ ).

\section{Traitement Produit Quantité Oxyde ou Quantité élément}

$\begin{array}{lcrcr}\text { Témoin } & \text { Aucun } & 0 & \text { Aucun } & 0 \\ \text { CaMg } & \mathrm{CaCO}_{3} & 1400 & \mathrm{CaO} & 784 \\ & \mathrm{CaO} & 1100 & \mathrm{CaO} & 1100 \\ & \mathrm{MgO} & 400 & \mathrm{MgO} & 400 \\ \text { NPKCaMg CaCO} & 1400 & \mathrm{CaO} & 784 \\ \mathrm{CaO} & 1100 & \mathrm{CaO} & 1700 \\ & \mathrm{MgO} & 400 & \mathrm{MgO} & 400 \\ \mathrm{KCl} & 250 & \mathrm{~K}_{2} \mathrm{O} & 160 \\ & \mathrm{Khosphate} & 445 & \mathrm{P}_{2} \mathrm{O}_{5} & 200 \\ & \text { super triple } & & & \\ \mathrm{NH}_{4} \mathrm{NO}_{3} & 580 & \mathrm{~N} & 200\end{array}$

Souchier (1979), en utilisant en particulier la méthode de Rouiller et al (1980), pour déterminer la capacité d'échange cationique (CEC) et la garniture ionique du complexe adsorbant au $\mathrm{pH}$ du sol.

Les analyses des solutions ont été réalisées par spectrophotométrie ICP $(\mathrm{Si}, \mathrm{Al}, \mathrm{Fe}, \mathrm{Ca}, \mathrm{K}, \mathrm{Na}$, $\mathrm{Mg}, \mathrm{Mn}, \mathrm{P}$ et S) ou par colorimétrie $\left(\mathrm{NH}_{4}{ }^{+}, \mathrm{NO}_{3}{ }^{-}\right.$, $\mathrm{Cl}^{-}$) après filtration à $0,45 \mu$ des solutions brutes.

Les analyses de végétaux ont été réalisées après minéralisation par voie humide par spectrophotométrie ICP ( $\mathrm{P}, \mathrm{K}, \mathrm{Ca}, \mathrm{Mg}, \mathrm{Mn}, \mathrm{S}$ et $\mathrm{Al}$ ) ou par colorimétrie ( $\mathrm{N}$ total).

Le calcul du bilan entrées-sorties de l'écosystème a été effectué à partir des données suivantes.

- Les apports atmosphériques sont évalués en considérant que $100 \%$ de $\mathrm{N}, \mathrm{S}, \mathrm{Na}, \mathrm{Cl}, \mathrm{Al}$ et $\mathrm{Fe}$, $80 \%$ de $\mathrm{Ca}, 50 \%$ de $\mathrm{Mg}$ et $10 \%$ de $\mathrm{K}$ et $\mathrm{Mn}$ mesurés dans les pluviolessivats nets (éléments des pluviolessivats bruts - éléments de la pluie incidente) ont une origine atmosphérique (dépôts secs), le reste constitue la récrétion (lixiviation des éléments des cellules végétales par les eaux de pluie) (Probst et al, 1990a).

- Les entrées par altération sont évaluées par un calcul complexe détaillé par Bonneau et al (1992).

- Les immobilisations sont évaluées à partir des calculs de minéralomasse.

- Les pertes par drainage sont évaluées à partir des concentrations mesurées des solutions et d'un bilan hydrique théorique puisque les données mesurées par les plaques ne peuvent être directement utilisées. Le drainage est calculé par l'équation générale décrite par Aussenac (1975):

$$
\text { Drainage }(\mathrm{mm})=P-l n-E T R \pm \Delta s
$$

Avec $P=$ précipitation incidente; $l n=$ interception par les cimes ; ETR = evapo-transpiration réelle ; $\Delta s=$ variation de la réserve hydrique du sol (évaluée à partir des courbes pF-humidité, établies en utilisant la méthode de Richards (1947).

Cependant, $P$ - In dans l'équation ci-dessus a été remplacé par le pluviolessivage total (égouttements des cimes + écoulements de tronc), parce qu'une estimation satisfaisante de ces paramètres est difficile à obtenir par mesure directe. Les données de l'évapo-transpiration potentielle (ETP Penman) utilisées pour le site du Bonhomme, ont été mesurées à Aubure (Granier, 1990, données non publiées), les conditions écologiques entre le site du Bonhomme et celui d'Aubure étant voisines. Le passage à l'ETR (évapotranspiration réelle) nécessite la mesure de la transpiration du peuplement et celle de l'évaporation directe. Les résultats obtenus sur le site voisin d'Aubure (Granier, 1990, données non publiées) ont été utilisés: la transpiration par le peuplement et l'évaporation directe à partir du sol ont donc été estimées respectivement à $35 \%$ et $5 \%$ de l'ETP Penman. Afin d'effectuer un bilan par horizon, nous avons fait l'hypothèse que le peuplement prélevait l'eau au prorata de la densité radiculaire des arbres: $60 \%$ de l'eau sont supposés être prélevés entre $0-15 \mathrm{~cm}, 20 \%$ pour chacun des niveaux $15-30$ et $30-60 \mathrm{~cm}$. Pendant les mois d'hiver, quand la température moyenne est $\leq 0$, l'évapo-transpiration (ETR) est considérée comme nulle.

L'équation de bilan hydrique est donc la suivante:

Drainage $(\mathrm{mm})=$ pluviolessivage total $-E T R \pm \Delta S$

\section{RÉSULTATS ET DISCUSSION}

\section{Effets des traitements sur les peuplements}

Le peuplement se caractérise par une mauvaise venue et une faible production en liai- 
son avec un sol pauvre et une situation d'altitude en zone de crête : la hauteur moyenne d'environ $20 \mathrm{~m}$ à 70 ans le place dans la classe 5 de la table de production de l'épicéa commun dans le nord-est de la France de Decourt (1973). Les arbres présentent souvent des doubles cimes dues au givre.

En 1986, l'état sanitaire des peuplements était caractérisé par un niveau de jaunissement moyen compris entre 25 et $50 \%$ et un taux moyen de défoliation voisin de $40 \%$. La revitalisation a été très importante et très rapide puisque les symptômes de dépérissement avaient nettement régréssé 2 ans après l'application des traitements et qu'ils ont pratiquement disparu au bout de 5 ans. L'état sanitaire du peuplement témoin fluctue au cours du temps mais reste toujours très mauvais (tableau III).

Les analyses foliaires (non présentées) indiquent un niveau critique d'alimentation en $\mathrm{Ca}$ (de 0,15 à $0,30 \%$ en fonction des années) et une carence en $\mathrm{Mg}$ (de 0,04 à $0,06 \%$ ) des arbres du peuplement témoin.
La nutrition des arbres en ces éléments s'est considérablement améliorée dans les 2 traitements (de 0,30 à $0,45 \%$ en $\mathrm{Ca}$ et de 0,09 à $0,11 \%$ en $\mathrm{Mg}$ dans les 2 traitements $\mathrm{CaMg}$ et NPKCaMg) ; la nutrition en $\mathrm{N}$ et $\mathrm{K}$ a peu évolué et celle en $P$ s'est améliorée dans le traitement NPKCaMg. Bonneau (1993) fait le point sur cet aspect pour l'ensemble des peuplements de sapin et d'épicéa commun traités dans les Vosges.

La biomasse totale ligneuse sur pied de $200 \mathrm{t} \mathrm{ha}^{-1}$ est faible si on la compare aux $400 \mathrm{t} \mathrm{ha}^{-1}$ de la pessière bienvenante de Gemaingoutte (Vosges), située cependant à $650 \mathrm{~m}$ d'altitude (Ranger et al, 1992). La production moyenne des peuplements est faible et de l'ordre de $6 \mathrm{~m}^{3} \cdot \mathrm{ha}^{-1} \cdot \mathrm{an}^{-1}$. L'effet des traitements est limité sur la production, avec une amélioration sensible sur l'accroissement en circonférence $\left(\mathrm{C}_{130}\right)$ pour le peuplement chaulé (CaMg). Compte tenu de la précision des mesures de circonférence, on ne peut évaluer que grossièrement cette augmentation de production.

Tableau III. Caractéristiques du peuplement et effets des traitements sur le dépérissement.

\begin{tabular}{|c|c|c|c|}
\hline Paramètre & Témoin & $\mathrm{CaMg}$ & NPKCaMg \\
\hline Circonférence moyenne en $1990(\mathrm{~mm})$ & 1036 & 1093 & 1050 \\
\hline Accroissement courant (mm) & 9 & 12,3 & 9 \\
\hline Hauteur moyenne $(\mathrm{m})$ & 20 & 20 & 20 \\
\hline \multicolumn{4}{|l|}{ Symptômes de dépérissement } \\
\hline $\begin{array}{ll}\text { Défoliation (\%) } & 1986\end{array}$ & 38 & 24 & 35 \\
\hline 1987 & 39 & 17 & 17 \\
\hline 1989 & 38 & 17 & 15 \\
\hline 1990 & 30 & 3 & 6 \\
\hline \multicolumn{4}{|l|}{ Jaunissement* ${ }^{*}$} \\
\hline 1986 & 2,00 & 1,70 & 1,80 \\
\hline 1988 & 1,52 & 0,17 & 0,32 \\
\hline 1989 & 1,87 & 0,08 & 0,64 \\
\hline 1990 & 2,00 & 0,04 & 0,00 \\
\hline
\end{tabular}

Les mesures d'accroissements et les notations de l'état sanitaire ont été effectuées sur 24 arbres par traitement. * Critères de notation par arbre : note 0 (de $0 \%$ et 10\%); note 1 (de $10 \%$ à 25\%) ; (\% d'aiguilles jaunes) note 2 (de $26 \%$ à $50 \%$ ); note $3(>60 \%)$. 
Dans le traitement CaMg on peut estimer l'augmentation de croissance à 1,5 à $2 \mathrm{~m}^{3} \cdot \mathrm{ha}^{-1} \cdot \mathrm{an}^{-1}$, ce qui est assez faible en valeur absolue mais élevé en valeur relative (Bonneau, communication personnelle). Une étude plus précise, comme celle réalisée sur sapin par Lebourgeois (1991), va être effectuée sur les essais vosgiens, de façon à préciser les effets des traitements sur la production. Belkacem et al (1992) ont évalué à $20 \%$ le gain de production de biomasse après chaulage d'un peuplement d'épicéa de 60 ans dans les Ardennes primaires.

Les restitutions par les litières sont les plus élevées dans le traitement témoin qui perd toujours plus d'aiguilles que les peuplements traités ; ces derniers étant en phase de reconstitution de leur masse foliaire.

Une évaluation du prélèvèment indique une efficience médiocre des éléments nutritifs et une faible différence entre les traitements (tableau IV).

\section{Effets des traitements sur les sols}

En ce qui concerne l'humus, le chaulage a provoqué une évolution très visible morphologiquement ; l'amélioration est notable, du moder dans le témoin, l'humus évolue vers le moder-mull dans le traitement $\mathrm{CaMg}$ et vers le mull oligotrophe dans le traitement NPKCaMg (observations après 4 ans d'application des traitements). Cela se traduit par une diminution notable de l'épaisseur de la litière fraîche et de la couche $\mathrm{H}$ (déjections), ainsi que par une apparition de structure d'origine biologique dans l'horizon $A_{1}$. En revanche, le rapport $C / N$ des humus, qui rend compte de la disponibilité de l'azote a peu évolué dans les traitements. Les données bibliographiques de l'effet du chaulage sur la minéralisation de l'azote organique sont très variables, voire contradictoires : effets positifs selon Siebt et Wittich (1997) et Schierl et Kreutzer (1989) ou négatifs selon Matzner et al (1985) et
Deromé et Pätilä (1990). Selon Nömmick (1979), la minéralisation des litières augmente avec le chaulage quand le $\mathrm{C} / \mathrm{N}$ de la couche $\mathrm{F} / \mathrm{H}$ est inférieur à 30 , l'inverse est observé quand ce $\mathrm{C} / \mathrm{N}$ devient supérieur à 30. Cette règle semble cependant souffrir des exceptions (Anderson et Persson, 1988).

Les résultats présentés au tableau $V$ montrent que les effets du chaulage sont majoritairement et significativement limités à la surface du sol (Mohamed, 1992 ; Mohamed et al, 1993). L'hétérogénéité des sols est partiellement en cause dans ce résultat. Ces effets sont classiques: augmentation du $\mathrm{pH}$, des teneurs en $\mathrm{Ca}_{\text {éch }}$. et $\mathrm{Mg}_{\text {éch }}$, diminution de l'acidité d'échange et en particulier de $\mathrm{Al}_{\text {éch. }}$. La resaturation du sol en Ca est plus limitée aux horizons superficiels que celle en $\mathrm{Mg}$; elle pourrait avoir comme origine l'affinité relative de ces ions pour les anions organiques (Derome et Pätilä, 1990; Kinniburgh et al, 1976). On observe également que $\mathrm{K}$ est significativement plus faible dans les horizons de surface des traitements que dans celui du témoin. Ce phénomène observé par Curtin et Smillie (1983) et Messick et al (1984) dans des expériences de chaulage pourrait se traduire négativement sur la nutrition potassique des arbres.

Le traitement NPKCaMg a apparemment des effets moindres sur la neutralisation de l'acidité et sur la resaturation en $\mathrm{Ca}$ et $\mathrm{Mg}$ du sol que le chaulage seul. Cette différence pourrait avoir pour origine un "effet anion": $\mathrm{Cl}-, \mathrm{NO}_{3}{ }^{-}, \mathrm{SO}_{4}{ }^{2-}$ apportés par les fertilisants auraient servi ou servent encore de vecteur à la migration profonde des cations les plus mobiles. II apparaît que $\mathrm{Mg}$ est plus mobile que $\mathrm{Ca}$ dans ce contexte physicochimique. Derome (1988) signale la désorption préférentielle de $\mathrm{Mg}$ dans des sols de Finlande en voie d'acidification.

La pénétration relativement profonde des cations $\mathrm{Ca}$ et $\mathrm{Mg}$ dans le sol des 2 traitements, par rapport à ce qui est habituellement rapporté dans la littérature (Matzer et al, 1985 ; Anderson et Persson, 1988 ; 
Tableau IV. Biomasse, minéralomasse, immobilisation, restitutions par les litières et prélèvements moyens du peuplement dans les différents traitements.

\begin{tabular}{|c|c|c|c|c|c|c|c|}
\hline Biomasse & $N$ & $P$ & $K$ & $\mathrm{Ca}$ & $M g$ & $M n$ & $S$ \\
\hline
\end{tabular}

\begin{tabular}{|c|c|c|c|c|c|c|c|c|}
\hline \multicolumn{9}{|l|}{ Biomasse } \\
\hline Total tronc & 176,7 & 167,7 & 11,7 & 113,7 & 142,8 & 22,3 & 20,5 & 18,6 \\
\hline Branches & 23,5 & 152,0 & 28,0 & 97,0 & 68,0 & 23,6 & 15,2 & 8,7 \\
\hline Total épigé ligneux & 200,2 & 319,7 & 39,7 & 210,7 & 210,8 & 45,9 & 35,7 & 27,3 \\
\hline \multicolumn{9}{|l|}{ Litière $\left(\mathrm{kg} \mathrm{ha}^{-1} \mathrm{an}^{-1}\right)$} \\
\hline Témoin aiguilles & 3460 & 48,0 & 3,5 & 7,8 & 10,5 & 0,9 & 1,3 & 3,5 \\
\hline Témoin total & 4700 & 60,8 & 4,3 & 9,6 & 14,1 & 1,1 & 1,6 & 4,6 \\
\hline Traitement CaMg aiguilles & 2180 & 27,7 & 2,2 & 5,1 & 7,2 & 0,8 & 1,6 & 2,2 \\
\hline Traitement CaMg total & 3370 & 40,8 & 3,0 & 6,8 & 10,1 & 1,1 & 1,9 & 3,7 \\
\hline Traitement NPKCaMg aiguilles & 3000 & 39,2 & 3,5 & 8,1 & 9,8 & 1,1 & 2,4 & 2,8 \\
\hline Traitement NPKCaMg total & 4500 & 56,4 & 4,8 & 11,1 & 13,8 & 1,5 & 2,9 & 4,7 \\
\hline \multicolumn{9}{|l|}{ Immobilisation a } \\
\hline Total épigé ligneux & 3100 & 4,9 & 0,6 & 3,2 & 3,2 & 0,7 & 0,5 & 0,4 \\
\hline \multicolumn{9}{|l|}{ Prélèvement $\mathrm{b}$ moyen annuel } \\
\hline Traitement Témoin & & 53,0 & 4,1 & 28,8 & 15,0 & 2,1 & 2,3 & 3,9 \\
\hline Traitement $\mathrm{CaMg}^{*}$ & & 55,4 & 4,6 & 23,5 & 17,1 & 2,5 & 3,8 & 4,0 \\
\hline Traitement NPKC CaMg* & & 56,7 & 5,3 & 18,3 & 17,1 & 2,6 & 4,2 & 4,1 \\
\hline
\end{tabular}

a Accroissement moyen annuel $x$ Concentration moyenne du tronc ; $b$ Immobilisation + restitutions (par les litières et la récrétion); * le prélèvement des arbres dans les traitements doit être corrigé pour tenir compte de la reconstitution de la masse foliaire (on a arbitrairement fixé les retombées de litière d'aiguilles à $4 \mathrm{t} /$ ha et par an.

Derome et Pätilä, 1990 ; Matzner et Meiwes, 1990), peut s'expliquer par l'utilisation de produits solubles ( $c f$ teneur en $\mathrm{CaO}$ et $\mathrm{MgO}$ dans le tableau II).

\section{Éléments dans les solutions ; aspect qualitatif}

\section{Transfert dans les solutions gravitaires}

Données moyennes

pour les 3 années d'observation

La charge ionique des solutions augmente schématiquement de manière importante depuis les pluies jusqu'aux percolats des horizons organo-minéraux, puis elle diminue progressivement des horizons organominéraux jusqu'au ruisseau. On observe que le déficit de charge des balances ioniques est le plus important dans les horizons riches en matière organique. Une relation significative existe entre le déficit ionique des solutions gravitaires et le $\mathrm{C}_{\text {org }}$ total des horizons correspondants ( $r=0,85, n=12)$; cette relation tend à montrer que la participation des acides organiques à l'équilibre ionique des solutions gravitaires serait de l'ordre de $20 \%$ en moyenne. Les traitements et en particulier le traitement $\mathrm{CaMg}$ ont tendance à réduire la charge ionique des solutions gravitaires du sol (tableau VI). 
Tableau V. Effet du chaulage associé ou non à une fertilisation sur le $\mathrm{pH}$ et sur les éléments échangeables du sol ( $\mathrm{K}, \mathrm{Ca}, \mathrm{Mg}$ et acidité d'échange; résultats en meq. $\left.100 \mathrm{~g}^{-1}\right)$.

\begin{tabular}{|c|c|c|c|c|c|c|c|}
\hline \multirow[t]{2}{*}{ Paramètre } & \multirow[t]{2}{*}{ Traitement } & \multicolumn{6}{|c|}{ Horizon et profondeur $(\mathrm{cm})$} \\
\hline & & $\begin{array}{c}A_{1} \\
(0-7)\end{array}$ & $\begin{array}{c}A_{2} B h \\
(7-14)\end{array}$ & $\begin{array}{c}B h \\
(14-20)\end{array}$ & $\begin{array}{c}\text { Bs1 } \\
(20-35)\end{array}$ & $\begin{array}{c}B s 2 \\
(35-60)\end{array}$ & $\begin{array}{c}C \\
(>60)\end{array}$ \\
\hline \multicolumn{8}{|l|}{$\mathrm{pH}_{2} \mathrm{O}$} \\
\hline & témoin & $3,4 \mathrm{a}$ & 3,6 a & $3,8 \mathrm{a}$ & $4,1^{a}$ & $4,1^{a}$ & $4,4 \mathrm{a}$ \\
\hline & $\mathrm{CaMg}$ & $4,5 \mathrm{~b}$ & 3,9 b & 4,1 a & 4,2 a & 4,4 a & 4,4 a \\
\hline & NPKCaMg & 3,9 a & $3,9 \mathrm{~b}$ & 4,2 a & 4,3 a & 4,4 a & $4,5^{a}$ \\
\hline \multicolumn{8}{|c|}{$K^{+}$échangeable (meq $100 \mathrm{~g} \mathrm{~g}^{-1}$ ) } \\
\hline & témoin & $0,51 \mathrm{a}$ & $0,23^{a}$ & 0,19 a & $0,14 \mathrm{a}$ & $0,12^{a}$ & 0,12 a \\
\hline & $\mathrm{CaMg}$ & $0,31 \mathrm{~b}$ & $0,17 \mathrm{a}$ & $0,15 \mathrm{a}$ & $0,15 \mathrm{a}$ & 0,13 a & 0,13 a \\
\hline & NPKCaMg & $0,42 a b$ & $0,22 a$ & $0,18 \mathrm{a}$ & $0,16 a$ & 0,19 a & 0,19 a \\
\hline \multicolumn{8}{|c|}{$\mathrm{Ca}^{2+}$ échangeable (meq $100 \mathrm{~g}^{-1}$ ) } \\
\hline & témoin & 0,79 a & 0,17 a & 0,14 a & 0,12 a & $0,10^{a}$ & $0,08^{a}$ \\
\hline & $\mathrm{CaMg}$ & $11,77 b$ & 0,60 a & $0,31 \mathrm{a}$ & 0,17 a & $0,12 a$ & $0,15 \mathrm{~b}$ \\
\hline & NPKCaMg & $5,82 \mathrm{c}$ & 0,67 a & $0,32 \mathrm{a}$ & 0,21 a & $0,40^{a}$ & 0,07 a \\
\hline \multicolumn{8}{|c|}{$\mathrm{Mg}^{2+}$ échangeable (meq $100 \mathrm{~g}^{-1}$ ) } \\
\hline & témoin & $0,30^{a}$ & $0,10^{a}$ & $0,06^{a}$ & 0,05 a & $0,03^{a}$ & 0,02 a \\
\hline & $\mathrm{CaMg}$ & $3,13^{b}$ & $0,46^{b}$ & $0,31^{b}$ & $0,17^{b}$ & $0,12^{b}$ & $0,11^{b}$ \\
\hline & NPKCaMg & $1,24 a$ & 0,44 a & $0,22^{\circ}$ & $0,15 b$ & 0,140 & $0,06 a b$ \\
\hline \multicolumn{8}{|c|}{ Acidité échangeable $\left(\mathrm{H}^{+}+\mathrm{A}^{3+}\right.$, meq $\left.100 \mathrm{~g}^{-1}\right)$} \\
\hline & témoin & 12,74 a & 13,98 a & 15,30 a & $12,70^{a}$ & $10,67^{a}$ & $7,00 \mathrm{a}$ \\
\hline & $\mathrm{CaMg}$ & $3,58^{b}$ & $10,81 \mathrm{~b}$ & $10,60^{b}$ & $9,60 \mathrm{~b}$ & 7,52 a & 5,58 a \\
\hline & NPKCaMg & $6,53^{b}$ & $11,09 \mathrm{~b}$ & $9,60^{b}$ & $8,30^{b}$ & $8,10^{a}$ & 5,95 a \\
\hline
\end{tabular}

Les lettres indiquent si les traitements sont semblables (même lettre) ou différents au seuil de $5 \%$ (lettre différente) pour un paramètre.

Les précipitations incidentes ont une composition chimique dominée par $\mathrm{SO}_{4}{ }^{2-}$, $\mathrm{N}\left(55 \%\right.$ de $\mathrm{NO}_{3}-$ et $45 \%$ de $\left.\mathrm{N}-\mathrm{NH}_{4}{ }^{+}\right), \mathrm{H}^{+}$ $\left(\mathrm{pH}\right.$ moyen $=4,52$ ) et divers cations, $\mathrm{Ca}^{2+}$, $\mathrm{Mg}^{2+}$ et $\mathrm{K}+$. Ces concentrations ont peu varié pendant les 3 années d'observation; les apports sont légèrement plus élevés au printemps et en été que pendant le reste de l'année.

Les modifications des concentrations moyennes des solutions sur les 3 années d'observation, au cours de leur transfert dans l'écosystème, sont résumées au tableau VII et illustrées par la figure 1.
Dans le traitement témoin, les pluviolessivats $s$ / (lessivage des cimes et ruissellement de troncs) sont notablement enrichis en tous éléments par rapport aux pluies incidentes, principalement par dépôt sec (cas de $N, S, C a$ ) et par lixiviation des éléments des cellules végétales (cas de $\mathrm{K}, \mathrm{Mn}$ et $\mathrm{Mg}$ ). Leur $\mathrm{pH}$ a considérablement baissé par rapport aux pluies incidentes $(\mathrm{pH}=4,1$ dans le pluviolessivat de cime et 3,7 dans le ruissellement de tronc).

Dans I'horizon holorganique, les solutions s'enrichissent à nouveau en particulier en $\mathrm{N}^{-\mathrm{NO}_{3}}{ }^{-}, \mathrm{Ca}^{2+}$ et $\mathrm{H}^{+}$. À $15 \mathrm{~cm}$ de pro- 
Tableau VI. Balance ionique et carbone total dissous des solutions gravitaires du témoin et des traitements CaMg et NPKCaMg.

\begin{tabular}{|c|c|c|c|c|c|}
\hline $\begin{array}{l}\text { Traitements } \\
\text { et niveaux }\end{array}$ & $\begin{array}{l}\sum \text { cations } \\
\left(\mu e ́ q \vdash^{-1}\right)\end{array}$ & $\begin{array}{l}\sum \text { anions } \\
\left(\mu e ́ q \vdash^{-1}\right)\end{array}$ & $\begin{array}{l}\sum \text { cations- } \\
\sum \text { anions } \\
\left(\mu e ́ q t^{-1}\right)\end{array}$ & $\begin{array}{l}\text { Déficit } \\
(\%)\end{array}$ & $\begin{array}{c}\text { Carbone } \\
\text { organique } \\
\left(\left.m^{-1}\right|^{-1}\right)\end{array}$ \\
\hline \multicolumn{6}{|l|}{ Témoin } \\
\hline Pluie externe & 95,1 & 95,0 & 0,1 & 0,0 & 5,4 \\
\hline Pluviolessivats & 295,7 & 282,2 & 13,5 & 5,0 & 12,0 \\
\hline Colliers & 866,3 & 711,1 & 155,2 & 18,0 & 46,7 \\
\hline Litière & 473,9 & 386,0 & 88,0 & 19,0 & 34,0 \\
\hline $15 \mathrm{~cm}$ & 799,0 & 633,8 & 165,3 & 21,0 & 19,0 \\
\hline $30 \mathrm{~cm}$ & 600,6 & 471,0 & 129,6 & 22,0 & 17,6 \\
\hline $60 \mathrm{~cm}$ & 633,8 & 578,7 & 55,0 & 9,0 & 7,9 \\
\hline \multicolumn{6}{|l|}{$\mathrm{CaMg}$} \\
\hline Pluviolessivats & 215,3 & 214,4 & 0,9 & 0,0 & 4,1 \\
\hline Colliers & 819,4 & 735,7 & 83,7 & 10,0 & 36,3 \\
\hline Litière & 424,9 & 289,6 & 135,3 & 32,0 & 34,9 \\
\hline $15 \mathrm{~cm}$ & 537,0 & 373,9 & 163,1 & 30,0 & 33,8 \\
\hline $30 \mathrm{~cm}$ & 345,3 & 312,9 & 32,3 & 9,0 & 11,8 \\
\hline $60 \mathrm{~cm}$ & 397,7 & 405,4 & $-7,6$ & 2,0 & 6,8 \\
\hline \multicolumn{6}{|l|}{ NPKCaMg } \\
\hline Pluviolessivats & 243,5 & 242,6 & 0,9 & 0,0 & 4,5 \\
\hline Colliers & 657,0 & 592,7 & 64,2 & 10,0 & 30,2 \\
\hline Litière & 444,4 & 294,0 & 150,4 & 34,0 & 30,9 \\
\hline $15 \mathrm{~cm}$ & 843,6 & 525,6 & 318,0 & 38,0 & 44,6 \\
\hline $30 \mathrm{~cm}$ & 482,3 & 353,3 & 129,0 & 27,0 & 21,2 \\
\hline $60 \mathrm{~cm}$ & 577,2 & 571,2 & 6,0 & 1,0 & 8,8 \\
\hline
\end{tabular}

fondeur, $\mathrm{N}-\mathrm{NO}_{3}{ }^{-}, \mathrm{Al}^{3+}$ et $\mathrm{H}^{+}$augmentent fortement tandis qu'à $30 \mathrm{~cm}$ ces mêmes ions décroissent de manière très importante. $\mathrm{N}-\mathrm{NO}_{3}$ - et $\mathrm{Al}^{3+}$ augmentent à nouveau à $60 \mathrm{~cm}$.

Les solutions sont très acides $(\mathrm{pH}$ moyen $=3,78$ à $15 \mathrm{~cm}$ et 4,25 à $60 \mathrm{~cm}$ ), riches en $\mathrm{Al}^{3+}$ et pauvres en $\mathrm{Ca}^{2+}$ et $\mathrm{Mg}^{2+}$; $\mathrm{Cl}^{-}$semble transféré sans changement dans les divers horizons et $\mathrm{SO}_{4}{ }^{2-}$ prend une importance relative quand la concentration en $\mathrm{N}-\mathrm{NO}_{3}$ - diminue à la base du profil.

$\mathrm{N}-\mathrm{NO}_{3}{ }^{-}$et la majorité des cations sont positivement corrélés, mais, compte tenu de la valeur absolue des concentrations, la corrélation entre $\mathrm{N}-\mathrm{NO}_{3}-$ et $\mathrm{Al}{ }^{3+}$ est la plus intéressante $(r=0,96, n=34 ; r=0,67, n=$ 36 ; et $r=0,75, n=28$ à 15,30 et $60 \mathrm{~cm}$ de profondeur respectivement). Malgré leur fluctuation importante au cours de leur transfert dans le sol, ces éléments dominent le chimisme des solutions gravitaires dans tous les horizons. Compte tenu du $\mathrm{pH}$, une part importante de l'aluminium est sous forme $\mathrm{Al}^{3+}$; de plus, seulement $20 \%$ de cet Al est complexé par la matière organique (déterminé par la méthode de Driscoll, 1984).

Les solutions récoltées à la base du sol sont acides et ne se neutralisent que dans le sous-sol. Cette acidité est neutralisée progressivement dans l'arène granitique puisque les plaques lysimétriques installées 


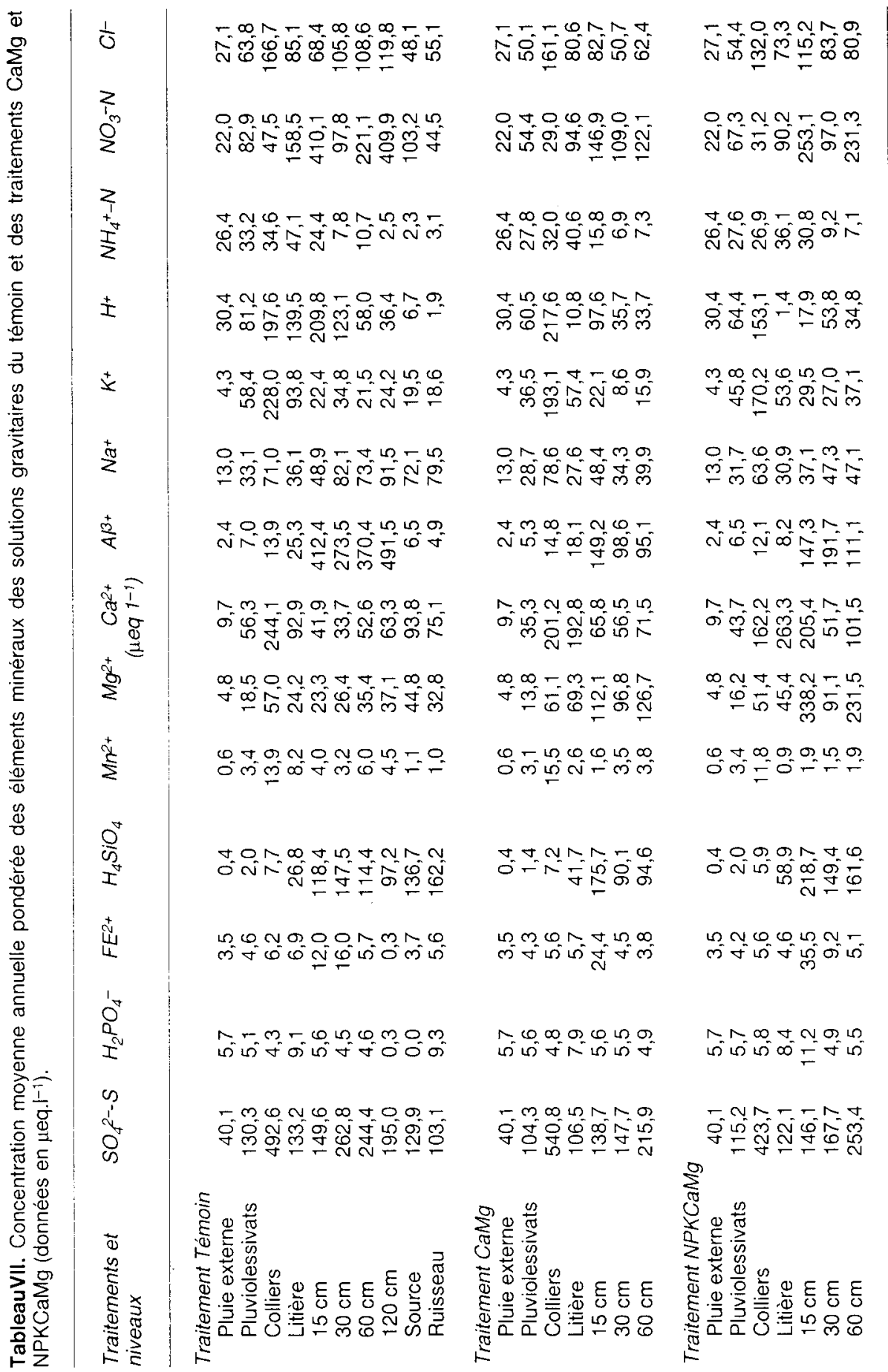



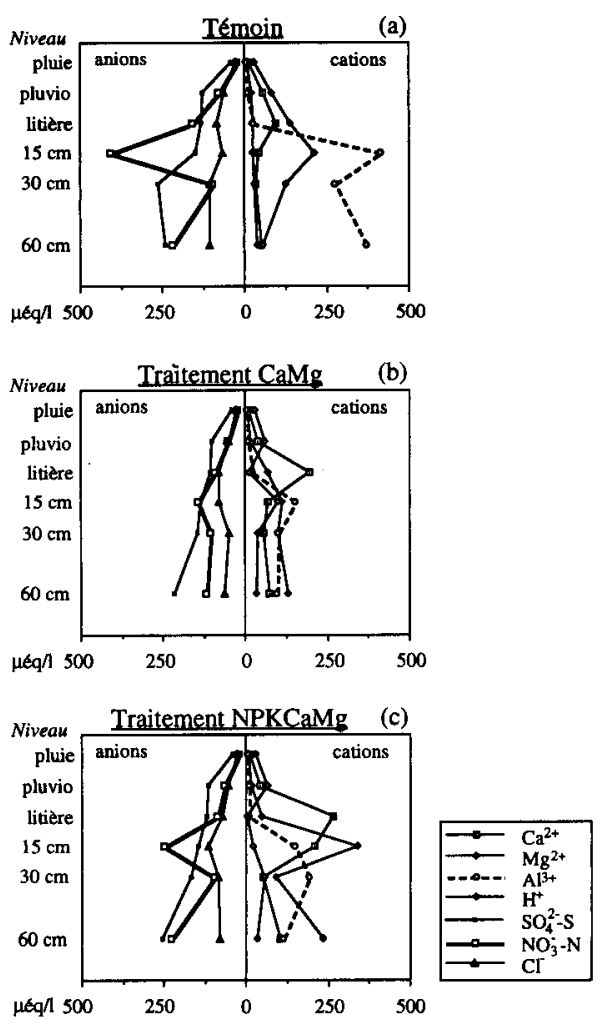

Fig 1. Distribution moyenne des anions et des cations dans les solutions gravitaires des diffèrents horizons du sol du témoin (a) et des traitements CaMg (b) et NPKCaMg (c) (données en ueq. $\left.\right|^{-1}$ ).

à 1,20 $\mathrm{m}$ de profondeur fournissent des solutions encore acides ( $\mathrm{pH}=4,4)$ et de composition peu différente de celle observée à $60 \mathrm{~cm}$ de profondeur.

Les eaux de source sont faiblement acides ( $\mathrm{pH}$ moyen $=5,2$ ), elles sont encore relativement chargées en nitrates (qui a cependant diminué de moitié par rapport à $60 \mathrm{~cm}$ ) mais très appauvries en aluminium. La neutralisation de l'acidité s'est faite aux dépens des minéraux altérables de l'arène libérant $\mathrm{Al}, \mathrm{Ca}$ et $\mathrm{Mg}$. Al est insolubilisé compte tenu $\mathrm{du} \mathrm{pH}$ : dans ces conditions l'électroneutralité de la solution est réalisée en ce qui concerne les cations essentiellement par Ca et Mg.

Les eaux du ruisseau drainant le bassin versant sont très faiblement acides $(\mathrm{pH}=$ 5,7 ) et leur concentration en tous éléments a baissé par rapport aux eaux de source. En valeur absolue, elles recèlent des teneurs en sulfates et nitrates et une acidité relativement élevées traduisant un bassin versant ayant des difficultés à neutraliser l'acidité du système comme c'est souvent le cas sur les roches mères acides des Vosges (Probst et al, 1990b).

L'effet des traitements sur les solutions gravitaires figure également au tableau VII et à la figure 1.

On n'observe que peu de différences significatives entre les compositions chimiques des pluviolessivats des traitements et du témoin ; c'est cependant le cas pour $\mathrm{Ca}^{2+}, \mathrm{K}^{+}$et $\mathrm{Cl}^{-}$du traitement $\mathrm{CaMg}$ qui se singuralise par les valeurs les plus faibles en ces éléments. La récrétion, serait la plus élevée dans le traitement témoin dépérissant, ce qui peut s'expliquer par une plus forte perméabilité cuticulaire dans ce traitement.

Dans les eaux gravitaires du sol du traitement $\mathrm{CaMg}$, les concentrations de $\mathrm{N}-\mathrm{NO}_{3}{ }^{-}$, $\mathrm{SO}_{4}{ }^{2-}, \mathrm{Al}^{3+}$ et $\mathrm{H}^{+}$diminuent considérablement par rapport au témoin ; à l'opposé celles de $\mathrm{Ca}^{2+}$ et $\mathrm{Mg}^{2+}$ augmentent nettement. Le pH des solutions reste malgré tout fortement acide $(4,0$ et 4,5 respectivement à 15 et $60 \mathrm{~cm}$ de profondeur) et l'acidité du système (quelle qu'en soit l'origine) n'est pas neutralisée dans le sol. Le rôle de l'ion nitrate a diminué par rapport à ce qu'il était dans le traitement témoin bien que les corrélations entre nitrates et cations restent supérieures à celles entre sulfates et cations.

Le traitement NPKCaMg a le même effet relatif, mais les concentrations en $\mathrm{N}-\mathrm{NO}_{3}{ }^{-}$, $\mathrm{Ca}^{2+}$ et $\mathrm{Mg}^{2+}$ y sont plus élevées que dans le traitement CaMg. 
Les 2 traitements neutralisent très significativement l'acidité de la solution du sol (et en particulier sa composante aluminique) qui se trouve enrichie en cations basiques par rapport à la solution du sol témoin. Les modifications sont nettes sur tout le profil de sol.

Le chaulage semble réduire la nitrification nette; l'association d'une fertilization aurait réactivé le cycle de l'azote. En effet, les teneurs en $\mathrm{N}_{-} \mathrm{NO}_{3}-$ observées dans le traitement NPKCaMg peuvent difficilement être attribuées à l'effet direct des fertilisants azotés solubles apportés plusieurs années auparavant. Dans ce traitement, le flux de nitrate entraîne une forte lixiviation de $\mathrm{Mg}^{2+}$.

Le chimisme des solutions gravitaires passe d'une dominance ionique $\mathrm{N}$ $\mathrm{NO}_{3}-/ \mathrm{Al}{ }^{3+}$ dans le témoin à une dominance $\mathrm{N}-\mathrm{NO}_{3}-/ \mathrm{Mg}^{2+}$ et $\mathrm{Al}^{3+}$ dans le traitement $\mathrm{CaMg}$ et $\mathrm{N}-\mathrm{NO}_{3}-/ \mathrm{Mg}^{2+}$ et $\mathrm{Ca}^{2+}$ dans le traitement NPKCaMg.

\section{Variabilité interannuelle}

de la concentration ionique des solutions

L'observation la plus remarquable concerne la décroissance importante de la concentration des solutions gravitaires en $\mathrm{N}-\mathrm{NO}_{3}{ }^{-}$ au cours des 3 années d'observation. Cette décroissance est la plus nette dans le témoin où elle est significativement corrélée à la diminution de la concentration en $\mathrm{Al}^{3+}$ (fig 2a) ; dans les 2 parcelles traitées, elle est corrélée à la diminution de concentration en $\mathrm{Mg}^{2+}$ (fig 2b).

L'origine de cette décroissance de la concentration en nitrates n'est pas liée aux apports atmosphériques beaucoup moins concentrés et plus ou moins constants au cours de la période d'observation. II semble difficile d'admettre comme explication le seul effet d'un artefact lié au système expérimental. Celui-ci n'a pas conduit à des tendances similaires dans d'autres situations, de plus un délai de 6 mois a été respecté entre l'installation et la première collecte de solutions. On peut faire l'hypothèse que l'on a observé un flux exceptionnel de nitrates lié à la minéralisation des chutes abondantes de litière après la défoliation des années 980. La relative amélioration naturelle des peuplements concourt également à la diminution de la concentration des nitrates en solution, ceux-ci étant absorbés en plus grande quantité par les arbres. Ces tendances singulières ne sont pas exceptionnelles sur de telles durées d'observation; les rares études portant sur plus de 15 ans d'observation (Driscoll et al, 1989) permettent de le vérifier.

\section{Variabilité saisonnière}

de la concentration ionique des solutions

Elle peut être liée à plusieurs facteurs :

- la variabilité des apports atmosphériques qui tendent sur la période considérée à être plus faibles en hiver; quelques variations singulières ont été enregistrées, comme par exemple les apports élevés du printemps 1991 ;

- la variation de la minéralisation au cours de l'année, qui se traduit par une augmentation des concentrations pendant la saison de végétation, particulièrement nette sous les horizons holorganiques;

- le prélèvement par la végétation ;

- les fluctuations des paramètres climatiques (pluviométrie et température) qui peuvent se traduire par des phénomènes de dilution/concentration ou des variations dans la vitesse de minéralisation.

- la vitalité du peuplement influe sur le prélèvement à la fois par la quantité prélevée et la durée du prélèvement dans le temps ; les arbres revitalisés tendraient à prélever plus longtemps en automne.

La concentration des eaux gravitaires est le résultat net de l'interaction entre ces facteurs dont l'effet individuel ne pourra pas être identifié facilement dans ce type d'approche. De plus, les eaux gravitaires n'ont pas strictement la même composition que 
$\operatorname{\mu eg} / 1^{12}$

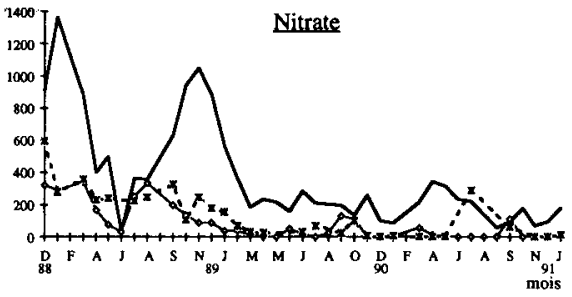

meql 1200

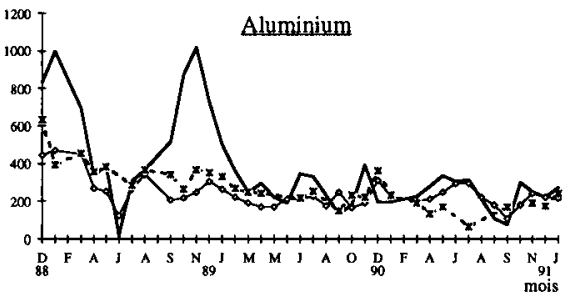

нeqh ${ }^{\infty}$
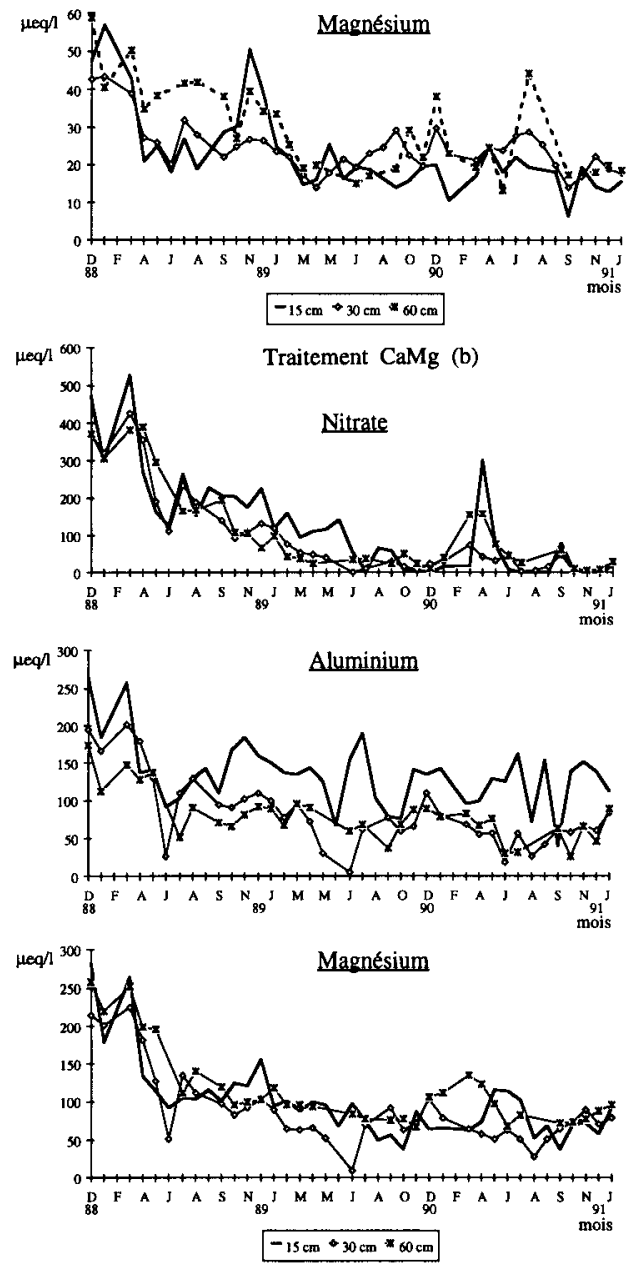
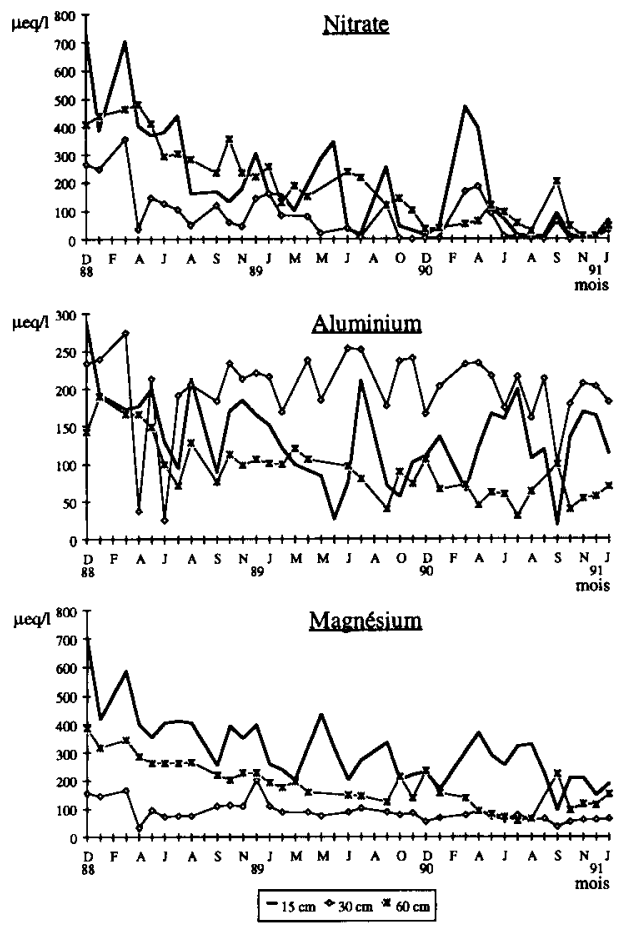

Fig 2. Évolution au cours des 3 années d'observations des teneurs en quelques éléments des solutions gravitaires des sols du témoin (a), et des traitements CaMg (b) et NPKCaMg (c). 
les solutions "capillaires" qui résultent de réactions tendant vers l'équilibre et autorisant une meilleure identification du fonctionnement du sol et de la qualité des solutions à partir desquelles les arbreś s'alimentent (Ranger et al, 1993).

Dans ce site acide les fluctuations saisonnières des concentrations en nitrates des eaux gravitaires soulèvent les problèmes suivants, centrés sur le fonctionnement du cycle de l'azote qu'il sera nécessaire d'étudier en détail :

- Les concentrations élevées des nitrates en hiver résultent-elles d'une minéralisation hivernale importante ou d'un simple déstockage à partir du compartiment capillaire ?

- La décroissance systématique des teneurs à $30 \mathrm{~cm}$ de profondeur en hiver, c'est-à-dire en dehors de tout prélèvement, résulte$\mathrm{t}$ - elle d'une réorganisation microbienne avec ou sans transfert sous forme organique, d'une perte par dénitrification ou de phénomènes purement physiques d'échanges entre compartiments gravitaires ou capillaires?

- Les teneurs à $60 \mathrm{~cm}$ de profondeur ontelles pour origine une minéralisation locale de la matière organique en déséquilibre dans l'écosystème actuel ou un transfert des horizons superficiels?

\section{Les bilans entrées-sorties d'éléments nutritifs établis dans les différents traitements, à l'échelle de l'écosystème}

Ces bilans sont établis à partir des données de flux d'apports (altération des minéraux de réserve, apports atmosphériques totaux) et de pertes (immobitisation dans la biomasse, drainage au-delà de la zone radiculaire) (Ranger et Bonneau, 1986). La comparaison des entrées et des sorties permet de juger l'état d'équilibre actuel de l'écosystème. Ces bilans n'indiquent que des tendances, dans la mesure où des incertitudes importantes existent sur l'évaluation des termes du bilan. Les plus fortes incertitudes résident sur le bilan hydrique mais surtout sur le flux d'altération que l'on ne sait pas mesurer de manière indépendante et qui ne peut qu'être évalué.

Le tableau VIII donne une évaluation moyenne sur 3 ans des flux traversant l'écosystème. Les apports atmosphériques totaux sont calculés à partir des pluviolessivats et les pertes par drainage sont directement issues des concentrations observées et du drainage théorique (cf Matériel et méthodes).

\section{Les bilans annuels}

Les bilans moyens pour les 3 années d'observation (tableau IX)

Dans le témoin, le bilan des élements majeurs de la fertilité minérale du sol est négatif, si on excepte le potassium.

Dans le traitement CaMg on a la même tendance relative, cependant les bilans de $\mathrm{Mg}$ et $\mathrm{Ca}$ sont plus fortement déficitaires que dans le témoin.

Dans le traitement NPKCaMg, tous les éléments (sauf $\mathrm{K}$ ) sont déficitaires ; les déficits de $\dot{\mathrm{Mg}}$, Ca ont fortement augmenté par rapport au traitement CaMg et a fortiori par rapport au témoin. Le bilan de $\mathrm{K}$ y est le moins nettement positif.

\section{Variabilité interannuelle}

des bilans entrées-sorties (tableau IX)

Dans le traitement témoin, le bilan d'azote fut très déficitaire en 1989, mais est devenu excédentaire en 1990 et 1991. Le bilan de Ca déficitaire en 1989 et 1990 est devenu excédentaire en 1991, celui de Mg est resté constamment déficitaire avec certes une diminution du déficit au cours du temps. Une corrélation évidente existe entre le déficit du bilan azoté et celui des cations. 


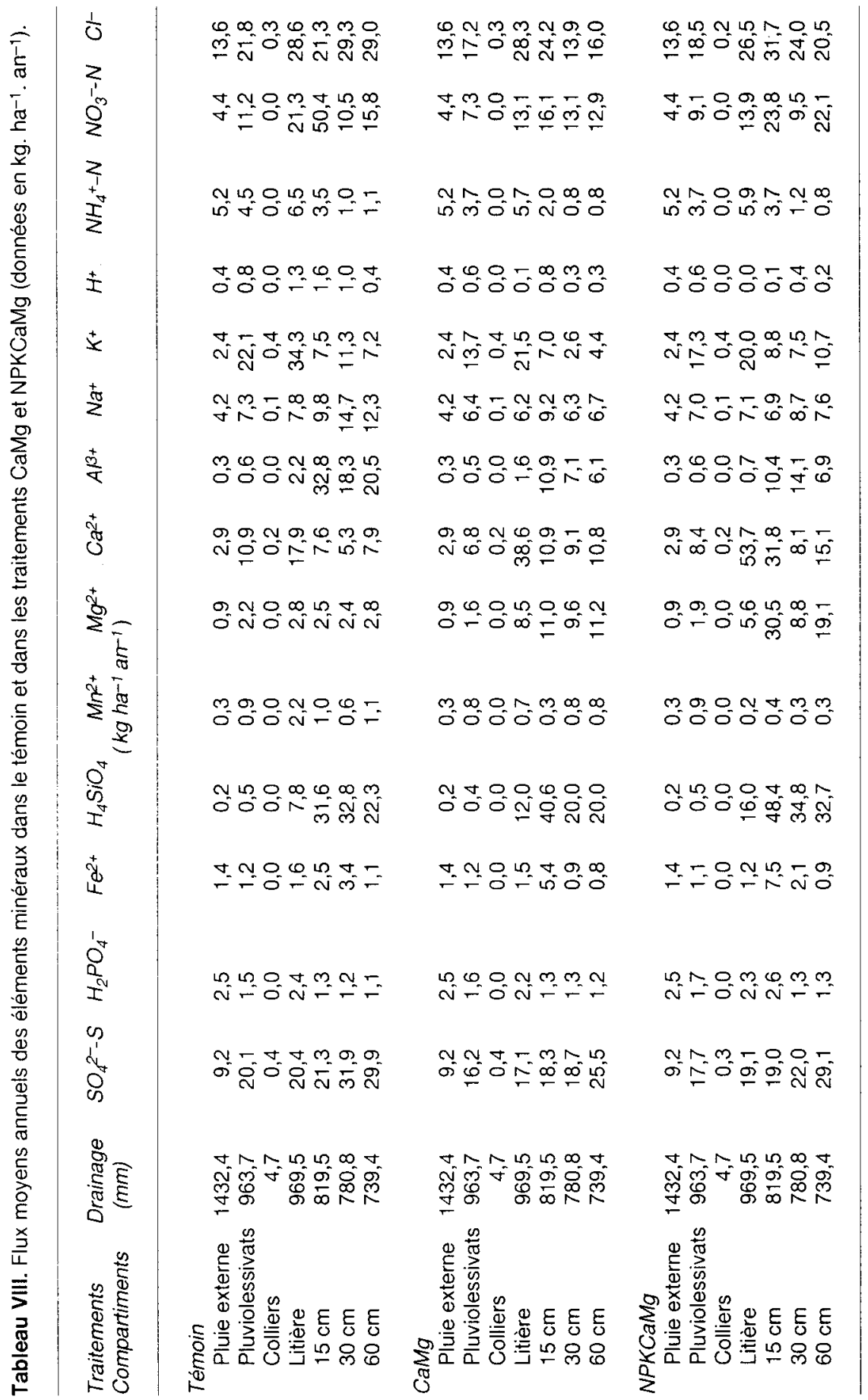


Tableau IX. Bilan entrées-sorties moyen annuel, et variation interannuelle, pour le témoin et pour les traitements CaMg, et NPKCaMg (données en kg. ha-1.an-1).

\begin{tabular}{|c|c|c|c|c|c|}
\hline & $M g$ & $\mathrm{Ca}$ & $K$ & $N$ & $s$ \\
\hline \multicolumn{6}{|l|}{ Tếtmoin } \\
\hline Précipitation incidente & 0,8 & 2,8 & 2,4 & 9,7 & 9,2 \\
\hline Dépôt sec & 0,7 & 6,7 & 2,0 & 6,0 & 11,4 \\
\hline Altération & 0,4 & 1,0 & 18,7 & & \\
\hline Immobilisation & 0,7 & 3,2 & 3,2 & 4,9 & 0,4 \\
\hline Drainage à $60 \mathrm{~cm}$ & 2,8 & 7,9 & 7,2 & 16,9 & 29,9 \\
\hline Entrées-sorties moyen & $-1,6$ & $-0,6$ & 12,7 & $-6,1$ & $-9,7$ \\
\hline Entrées-sorties 1989 & $-2,8$ & $-1,8$ & $+14,0$ & $-29,8$ & $-5,4$ \\
\hline Entrées-sorties 1990 & $-1,1$ & $-2,4$ & $+11,9$ & $+2,7$ & $-16,3$ \\
\hline Entrées-sorties 1991 & $-0,8$ & $+2,3$ & $+12,2$ & $+8,6$ & $-7,4$ \\
\hline \multicolumn{6}{|l|}{ Traitement CaMa } \\
\hline Précipitation incidente & 0,8 & 2,8 & 2,4 & 9,7 & 9,2 \\
\hline Dépôt sec & 0,4 & 3,4 & 1,2 & 1,4 & 7,4 \\
\hline Altération & 0,3 & 1,0 & 18,7 & & \\
\hline Immobilisation & 0,7 & 3,2 & 3,2 & 4,9 & 0,4 \\
\hline Drainage à $60 \mathrm{~cm}$ & 11,2 & 10,8 & 4,4 & 13,7 & 25,4 \\
\hline Entrées-sorties & $-10,4$ & $-6,8$ & 14,7 & $-7,5$ & $-9,2$ \\
\hline Entrées-sorties 1989 & $-16,4$ & $-7,3$ & $+14,7$ & $-23,9$ & $-6,5$ \\
\hline Entrées-sorties 1990 & $-7,6$ & $-7,3$ & $+15,5$ & $-0,1$ & $-13,0$ \\
\hline Entrées-sorties 1991 & $-6,9$ & $-5,6$ & $+13,8$ & $+1,8$ & $-8,1$ \\
\hline \multicolumn{6}{|l|}{ Traitement NPKCaMg } \\
\hline Précipitation incidente & 0,8 & 2,8 & 2,4 & 9,7 & 9,2 \\
\hline Dépôt sec & 0,6 & 4,7 & 1,5 & 3,1 & 9,0 \\
\hline Altération & 0,4 & 1,0 & 18,7 & & \\
\hline Immobilisation & 0,7 & 3,2 & 3,2 & 4,9 & 0,4 \\
\hline Drainage à $60 \mathrm{~cm}$ & 19,1 & 15,0 & 10,7 & 22,8 & 29,0 \\
\hline Entrées-sorties & $-18,0$ & $-9,7$ & $+8,7$ & $-14,9$ & $-11,2$ \\
\hline Entrées-sorties 1989 & $-26,3$ & $-13,0$ & $+6,6$ & $-33,7$ & $-14,1$ \\
\hline Entrées-sorties 1990 & $-16,1$ & $-11,4$ & $+9,7$ & $-11,3$ & $-13,0$ \\
\hline Entrées-sorties 1991 & $-11,7$ & $-5,0$ & $+10,0$ & $+0,5$ & $-6,7$ \\
\hline
\end{tabular}

Ces tendances générales s'observent également dans les 2 traitements;

\section{Les bilans entrées-sorties «saisonniers»}

\section{Les bilans saisonniers moyens}

II s'agit en faił de comparer les bilans établis pendant et hors période de végétation (tableau X).

Dans le témoin, pendant la dormance, les bilans de $\mathrm{N}, \mathrm{S}$ et $\mathrm{Mg}$ sont déficitaires, ceux de $\mathrm{Ca}$ sont excédentaires. Les apports atmosphériques servent essentiellement à nourrir le drainage profond (cela est particulièrement net pour $\mathrm{Ca}$ ). Le sol se désature en $\mathrm{Mg}$ pendant cette période, tandis que la tendance est plutôt à une resaturation, légère pour $\mathrm{Ca}$ et plus nette pour $\mathrm{K}$. Pendant la saison de végétation, les bilans de $\mathrm{Mg}, \mathrm{Ca}, \mathrm{S}$ et $\mathrm{N}$ sont négatifs, seul le bilan de $K$ est positif; les apports externes sont largement utilisés par les peuplements. Le prélèvement des arbres est le facteur principal de déficit du bilan. 


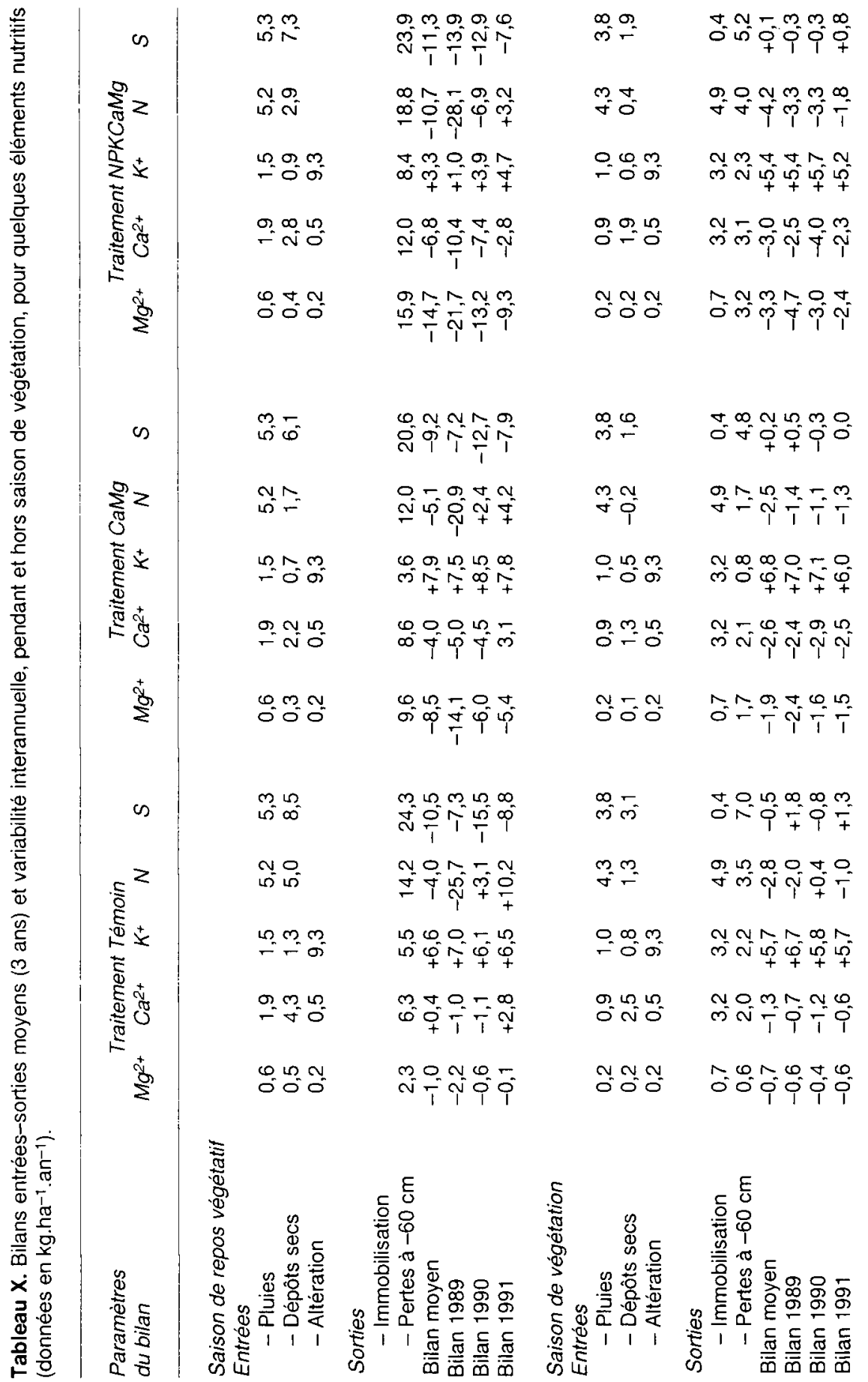


Dans les traitements, les mêmes tendances sont observées; les bilans de $\mathrm{Ca}$ et $\mathrm{Mg}$ sont plus négatifs que dans le témoin pour les 2 périodes. Les bilans de Mg sont plus négatifs que ceux de $\mathrm{Ca}$ pendant la saison de repos végétatif quand $\mathrm{N}_{-} \mathrm{NO}_{3}{ }^{-}$ n'est pas absorbé par les arbres. L'addition de fertilisants accroît le déficit de $\mathrm{Mg}$.

\section{La variabilité interannuelle des bilans saisonniers}

En 1989, dans le traitement témoin, les déficits sont les plus élevés en période de repos végétatif pour $\mathrm{N}, \mathrm{S}$, Ca et $\mathrm{Mg}$ seul $\mathrm{K}$ montre un bilan positif ; les mêmes tendances relatives s'observent dans les traitements $\mathrm{CaMg}$ et NPKCaMg avec cependant des pertes plus élevées que dans le témoin en $\mathrm{Ca}$ et $\mathrm{Mg}$.

En 1990, dans le traitement témoin, le bilan de $\mathrm{N}$ devient positif pendant la période de repos végétatif, le bilan de $S$ reste négatif et le déficit des bilans de $\mathrm{Ca}$ et $\mathrm{Mg}$ diminue très fortement. La même tendance relative s'observe dans les traitements pendant cette période. Les variations par rapport à 1989 sont beaucoup moins nettes pendant la saison de végétation quel que soit le traitement considéré.

En 1991, la réduction des déficits pendant la période de repos végétatif s'accentue, le bilan de $\mathrm{N}$ devient positif dans le témoin, les bilans de $\mathrm{Mg}$ et $\mathrm{S}$ restent négatifs. La même tendance relative existe dans les traitements où les bilans de $\mathrm{Ca}$ et $\mathrm{Mg}$ restent cependant assez nettement négatifs. Pendant la période de végétation, la variation des bilans par rapport à la moyenne est beaucoup plus limitée.

Au total, ce sont les variations du bilan de $\mathrm{N}$ et en particulier la tendance à la réduction du déficit en cet élément qui orientent celles du bilan des cations $\mathrm{Mg}$ et $\mathrm{Ca}$; le rôle de $S$ est moins net mais cet élément est le vecteur potentiel des cations quand $\mathrm{N}$ cesse de jouer ce rôle. Le bilan de $\mathrm{Mg}$ reste toujours négatif dans le témoin.

\section{CONCLUSIONS}

Le sol de l'écosystème non traité est caractérisé par une désaturation profonde du complexe absorbant et une forte acidité des solutions. Le fonctionnement actuel se traduit d'ailleurs par une acidification toujours active, sous l'influence, en particulier, du cycle de l'azote. L'origine des nitrates est mixte, externe, via les apports atmosphériques, mais surtout interne, malgré le pédoclimat fortement acide $\left(\mathrm{pH}_{\text {eau }}=3,25\right.$ en $\left.A_{1}\right)$. Il est probable que la défoliation observée au début des années 1980 a provoqué un flux exceptionnel de minéralisation, mais il est également possible que la matière organique du sol ne soit pas en équilibre avec le peuplement actuel. La production d'azote minéral dans les horizons profonds semble en être une preuve. $\mathrm{N}^{-\mathrm{NO}_{3}}$ - et $\mathrm{Al}^{3+}$ dominent la chimie des solutions gravitaires où la participation des anions organiques reste limitée (Mohamed, 1992). On remarque d'ailleurs que le fonctionnement actuel du sol n'est pas classique pour un sol de la série podzolique.

Le sol ne neutralise pas complètement l'acidité du milieu, ce qui n'est réalisé que dans l'arène profonde comme c'est le cas dans d'autres sites acides des Vosges. Le front d'acidification est situé relativement profondément dans le sous-sol puisqu'à 1,20 m les solutions sont toujours fortement acides.

Malgré la relative imprécision des bilans, les grandes tendances du fonctionnement de cet écosystème sont caractérisées par une désaturation permanente en ce qui concerne $\mathrm{Mg}$, qui est clairement l'élément nutritif limitant. Les fluctuations du cycle de l'azote se répercutent directement sur le bilan de cations, et peuvent conduire certaines années à un déficit en $\mathrm{Ca}$. Les bilans saisonniers montrent très clairement que 
les apports atmosphériques ne sont utiles au peuplement que lorsqu'ils sont apportés pendant la saison de végétation, ce qui avait déjà été mis en évidence par Bonneau et al (1991).

L'effet du chaulage est classique : il améliore la disponibilité de $\mathrm{Ca}$ et $\mathrm{Mg}$ et il diminue celle d'Al, toxique pour la végétation. Ces observations sont valables pour la phase solide et les solutions. La solubilité d'une partie des produits employés dans cette manipulation conduit à une migration de $\mathrm{Ca}$ limitée aux 20 premiers centimètres du sol, mais plus importante pour $\mathrm{Mg}$, qui paraît beaucoup plus mobile que $\mathrm{Ca}$ dans ce contexte. Le chaulage conduit à une diminution des teneurs en nitrates des eaux gravitaires qui semble indiquer une inhibition de la nitrification. Un travail complémentaíre prenant en compte les eaux capillaires a conduit à moduler cette affirmation (Ranger et al, 1993).

L'addition de fertilisants au chaulage conduit à une stimulation du cycle de l'azote qui entraîne une déperdition de cations. Cet effet n'est pas totalement négatif car il conduit à une resaturation plus profonde du sol, autorisant un approfondissement de l'enracinement, si cet effet est durable. En revanche, au niveau du seul bilan quantitatif, cet ajout de fertilisants qui n'apporte pas d'amélioration dans la revitalisation des peuplements par rapport au seul chaulage, conduit à un accroissement significatif des pertes par drainage, qui diminue par là même la durée d'efficacité du chaulage (on passerait très approximativement de 25 à 15 ans d'efficacité).

Pour ce qui concerne l'environnement, le chaulage conduit à une diminution du drainage d'Al sans augmentation des nitrates en solution, ce qui est très favorable; ; l'augmentation des pertes de nitrates liées à l'association de fertilisants est à prendre en compte. II semble que dans ce contexte cette adjonction de fertilisants ne soit globalement pas justifiée, ni pour les peuplements, ni pour l'environnement.
Quel que soit le traitement, la réponse des peuplements au chaulage est très favorable ; il améliore la disponibilité pour les plantes des éléments nutritifs majeurs dans les solutions et sur la phase solide, et il diminue le rôle toxique que peut avoir l'aluminium, comme le montre l'évolution de la valeur des rapports $\mathrm{Ca} / \mathrm{Al}$ et $\mathrm{Mg} / \mathrm{Al}$, dont l'augmentation de valeur ne peut être que favorable (Huttermann et Ulrich, 1984 ; Matzner et al, 1988).

L'amélioration de l'état sanitaire du peuplement ne se traduit pas dans cet écosystème par une augmentation très importante de la croissance des arbres; les mesures de circonférences (qui restent imprécises) indiquent que seul le traitement CaMg montre une augmentation de croissance. Ce point important qui peut in fine justitier économiquement le chaulage va être étudié sur l'ensemble des traitements vosgiens. L'amélioration du statut nutritif des arbres traités devrait, de plus, les rendre moins sensibles à de nouveaux stress, climatiques en particulier, qui pourraient conduire à des pertes de production sur les peuplements non traités.

Malgré la difficulté de cette approche écosystémique (hétérogénéité des systèmes naturels, variabilité interannuelle importante), cette expérience met en évidence le rôle que peut jouer le paramètre édaphique dans le dépérissement des peuplements forestiers.

\section{REMERCIEMENTS}

Ce travail a été subventionné par l'Office national des forêts. Nous remercions D Gelhaye, G Nourrisson et $B$ Pollier pour leur collaboration technique.

\section{RÉFÉRENCES}

Aussenac G (1975) Couverts forestiers et facteurs du climat : leurs interactions, conséquences ecophy- 
siologiques chez quelques résineux. Thèse Univ Nancy I, 234p

Andersson F, Persson T (1988) Liming as a measure to improve soil and tree condition in areas affected by air pollution. Nat Swedish Envir Board report 3518

Belkacem S, Nys C, Gelhaye D (1992) Effets d'une fertilisation et d'un amendement sur l'immobilisation d'éléments dans la biomasse d'un peuplement adulte d'épicéa commun (Picea abies L Karst). Ann Sci For 49, 235-252

Bonneau M (1993) Fertilisation sur résineux adultes (Picea abies Karst et Abies alba Mill) dans les Vosges : composition foliaire en relation avec la défoliation et le jaunissement. Ann Sci For 50, 159-175

Bonneau M, Fricker C (1985) Le dépérissement des forêts dans le massif vosgien : relations possibles avec la pot lution atmosphérique. Rev For Fr vol XXXVII, No spécial "Regards sur la santé de nos forêts", 105-126

Bonneau M, Landmann G (1988) De quoi la forêt est-elle malade ? La recherche 205, 1542-1553

Bonneau M, Souchier B (1979) Pédologie /l.Constituants et propriétés des sols. Masson, Paris, $459 \mathrm{p}$

Bonneau M, Dambrine E, Nys C, Ranger J (1991) Apports acides et cycles des cations : quelques réflexions à partir des dispositifs de Monthermé (Ardennes) et d'Aubure (Vosges). Sci Sol 29, 2, 125-145

Bonneau M, Landmann G, Adrian M (1992) La fertilisation comme remède au dépérissement des forêts en sol acide: essais dans les Vosges. Rev For Fr XLIV, 3, 207-224

Curtin D, Smillie GW (1983) Soil solution composition as affected by liming and incubation. Soil Sci Soc Am J 47, 701-707

Decourt N (1973) Tables de production pour les forêts françaises. ENGREF, $49 \mathrm{p}$

Derome J (1988) Cation mobility in forest soils. In: Air pollution and forest decline (J Bucher, I Bucher-Wallin, eds). Proc meeting IUFRO Interlaken, Switzerland 28 Oct, 399-401

Deromé J, Pätilä A (1990) Alleviation of forest soil acidification through liming. In: Acidification in Finland ( $\mathrm{P}$ Kauppi, $P$ Anttila, $K$ Kenttämies, eds). Springer-Verlag, Berlin, 1093-1115

Driscoll CT (1984) A procedure for the fractionation of aqueous aluminium in dilute acidic waters. Intern $J$ Environ Anal Chem 16, 267-283

Driscoll CT, Lickens GE, Hedin LO, Eaton JS, Bormann $\mathrm{FH}(1989)$ Changes in the chemistry of surface waters: 25 year results at the Hubbard Brook experiment forest (NH). Environ Sci Tech 23, 137-142

Hameurt $\mathrm{J}$ (1967) Les terrains cristallins et cristallophylliens du versant occidental des Vosges moyennes. Mém Serv Carte Géol Als Lorr 26

Huttermann A, Ulrich B (1984) Solid phase-solution-root interactions in soils subjected to acid deposition. Phil Trans Roy Soc London B 305, 353-368
Kinniburgh DG, Jackson ML, Syers JK (1976) Adsorption of alkaline earth, transition, and heavy metal cations by hydrous oxide gels of iron and aluminum. Soil Sci Soc Am J 40, 796-799

Lebourgeois $F$ (1991) Modifications observées à la suite d'une fertilisation dans diverses sapinières dépérissantes des Vosges. Étude phytoécologique et dendrochronologique. DEA, Univ Orsay

Matzner E, Khanna PK, Meiwes KJ, Ulrich B (1985) Effects of fertilization and liming on the chemical soil conditions and element distribution in forest soils. Plant Soil 87, 405-415

Matzner E, Meiwes KJ (1990) Effects of liming and fertilization on soil solution chemistry in north German forest ecosystems. WASP 54, 377-389

Matzner E, Blanck K, Stock HG (1988) Needle chlorosis pattern in relation to soil chemical properties in two Norway spruce (Picea abies Karst) forests of the German Harts mountains. In: Air pollution and forest decline (J Bucher, I Bucher-Wallin, eds). Proc Meeting IUFRO Interlaken, Switzerland 2-8 Oct 1988 195-199

Mehra OP, Jackson ML (1960) tron oxide removal from soil and clays by a dithionite citrate system buffered with sodium bicarbonate. Clays and Clay Minerals 7 . 317-327

Messick DL, Alley MM, Zelazny LW (1984) Movement of calcium and magnesium in ultisol from dolomitic limestone. Soil Sci Soc Am J 48, 1096-1101

Mohamed AD (1992) Rôle du facteur édaphique dans le fonctionnement biogéochimique de deux pessières vosgiennes : effet d'un amendement calci-magnésien. Thèse Univ Nancy I, $206 p$

Mohamed AD, Ranger J, Dambrine E, Bonneau M, Granier $A$ (1993) The effects of limestone plus NPK fertilization on the soil and mass balance of a spruce stand (Picea abies (L) Karst) in the Vosges mountains. For Ecol Manag 60, 291-310

Nömmick H (1979) The future role of liming in forestry. Skogs-o Landtbr-akad Tiskr Suppl 13, 31-37

Probst A, Dambrine E, Viville D, Fritz B (1990a) Influence of acid atmospheric inputs on surface water che mistry and mineral fluxes in a declining spruce stand within a small granitic catchment (Vosges massifFrance). J Hydrol 2, 116, 101-124

Probst A, Massabuau JC, Probst JL, Fritz B (1990b) Acidification des eaux de surface sous l'influence des précipitations acides : rôle de la végétation et du sustratum, conséquences pour les populations de truites; le cas des ruisseaux des Vosges. $C R$ Acad Sci Paris t 311, série II, 405-411

Ranger J, Bonneau M (1986) Effets prévisibles de l'intensification de la production et des récoltes sur la fertilité ces sols de forêts. Effets de la sylviculture. Rev For Fr XXXVIII, 2, 105-123

Ranger J, Cuirin G, Bouchon J, Colin-Belgrand M, Gelhaye D, Mohammed AD (1992) Biomasse et minéralomasse d'une plantation d'épicéa commun (picea 
abies Karst) de forte production dans les Vosges (France). Ann Sci For 49, 651-668

Ranger J, Discours D, Mohamed AD, Moares C, Dambrine E, Merlet D. Rouitler J (1993) Comparaison des eaux liées et des eaux libres étudiées dans les sols de trois pessières vosgiennes. Application à l'étude du fonctionnement actuel de des sols et conséquences pour l'état sanitaire des peuplements. Ann Sci For 50, 425-444

Richards LA (1947) Pressure membrane apparatus, construction and use. Agric Eng 28, 451-454

Rouiller J, Guillet B, Bruckert S (1980) Cations acides échangeables et acidités de surface, approche ana- lytique et incidences pédogénétiques. Sci Sol, AFES $2,161-175$

Schierl R, Kreutzer K (1989) Dolomitische Kalkung eines Fichtenbestandes aud saurer Parabraunerde: Auswirkungen auf Bodenchemie und vegetation. Kali Briefe 19, 417-423

Siebt G, Wittich W (1977) Ertragskundliche und bodenkundliche Ergebnisse langfristiger Kalkungsversuche im nord-und westdeutschen Bergland. Schriften d Forstl Fak d Univers, Göttingen Bd 50, 1-298

Tamura $T$ (1958) Identification of clay minerals from acid soils. J Soil Sci 9, 141-147 